\title{
Pediatric anterior cruciate ligament reconstruction
}

\author{
Mark O. McConkey • Davide Edoardo Bonasia • \\ Annunziato Amendola
}

Published online: 7 April 2011

(C) Springer Science+Business Media, LLC 2011

\begin{abstract}
An increasing number of anterior cruciate ligament (ACL) injuries are seen in children now than in the past due to increased sports participation. The natural history of ACL deficient knees in active individuals, particularly in children is poor. Surgical management of ACL deficiency in children is complex due to the potential risk of injury to the physis and growth disturbance. Delaying ACL reconstruction until maturity is possible but risks instability episodes and intra-articular damage. Surgical options include physeal-sparing, partial transphyseal and complete transphyseal procedures. This article reviews the management of ACL injured skeletally immature patients including the functional outcome and complications of contemporary surgical techniques.
\end{abstract}

M. O. McConkey

Department of Orthopaedics and Rehabilitation, University of Iowa Hospitals and Clinics,

2701 Prairie Meadow Drive,

Iowa City, IA 52242, USA

e-mail: mark_mcconkey@hotmail.com

D. E. Bonasia

Ist Department of Orthopaedics, CTO hospital,

University of Turin Medical School,

Via Lamarmora 26,

Torino 10128, Italy

e-mail: davidebonasia@virgilio.it

\author{
A. Amendola $(\bowtie)$ \\ Department of Orthopaedics and Rehabilitation, \\ UI Sports Medicine Center, \\ 2701 Prairie Meadow Drive, \\ Iowa City, IA 52242, USA \\ e-mail: ned-amendola@uiowa.edu
}

Keywords Pediatric · ACL - Anterior cruciate ligament . Immature $\cdot$ Prepubertal $\cdot$ Child · Skeletally immature $\cdot$ Knee $\cdot$ Physis · Physeal sparing · Transphyseal · Epiphyseal · Growth arrest · Growth disturbance · Over-the-top

\section{Introduction}

The knee is the most frequent site of musculoskeletal injury in the pediatric athlete [1]. The higher participation rate of children and adolescents in competitive sports has led to an increase in anterior cruciate ligament (ACL) injuries in the past decade [2]. Several retrospective reviews have reported that children and adolescents account for $0.5-3 \%$ of all ACL injuries [3-5]. The management of an ACL deficient child remains a controversial subject with debates surrounding operative timing and surgical technique.

If skeletally immature patients with ACL injury continue to be active in recreational play or competitive sports they risk chondral or meniscal injury which can lead to early osteoarthritis $[6,7]$. ACL reconstruction may improve knee function, avoid strict activity modification and reduce intraarticular injuries due to instability episodes $[6,8,9 \cdot 10,11]$.

A wide range of growth disturbances have been described in animal and clinical studies after ACL reconstruction [12]. The physes of the distal femur and proximal tibia are the primary contributors to lower extremity growth providing $70 \%$ and $60 \%$ of the growth of their respective bones [13]. Adolescents approaching skeletal maturity with limited growth remaining can be managed as adults with little risk of growth disturbance. However, drilling tunnels across open physes during ACL reconstruction can potentially injure the physis leading to premature closure, leg-length discrepancies and angular 
deformities. In an attempt to minimize the potential risk of growth disturbance surgeons have developed techniques to avoid physeal injury including: physeal-sparing techniques, partial transphyseal techniques and complete transphyseal techniques (Fig. 1).

Management of pediatric ACL injuries requires understanding of bone growth, mechanisms of physeal injury, as well as the natural history of delayed versus acute surgical management. The surgeon must be familiar with the unique surgical techniques and the risks and benefits associated with each.

\section{Clinical evaluation}

The evaluation of an injured child or adolescent can be challenging due to a lack of ability to articulate the circumstances of the injury or symptoms encountered since the injury. Discussion with a parent or other witness to the event can be invaluable. The physical examination can be more difficult due to difficulty in cooperating or fear of pain. Despite these limitations, an experienced clinician can expect an accurate assessment of the patient's injury with attention and patience [14].

The physician should elicit a detailed history of the injury including the mechanism of injury, feeling of a "snap" or "pop", the ability to bear weight or continue play after the event, and the severity and timing of any effusion that may have developed. The mechanism of injury most often is a non-contact pivoting motion on a fixed foot but the ACL can also be injured when the knee is hyperextended [15]. The onset of a hemarthrosis within hours of an injury is generally a manifestation of significant injury to the knee including ACL injury, tibial spine avulsion, meniscal injury, osteochondral fracture or patellar dislocation. Stanitski et al. [16] found that an acute knee effusion is associated with ACL injury in $47 \%$ of children aged 7 12 and $65 \%$ of adolescents aged 13-18. Luhmann [17] documented the pathology in 44 consecutive pediatric knees with an acute hemarthrosis and found a $29 \%$ incidence of ACL injury, 29\% incidence of meniscal tear, $25 \%$ of patellar dislocation, and $4 \%$ incidence of osteochondral fracture. Interestingly, $58 \%$ of effusions in girls were secondary to patellofemoral injury versus $12 \%$ in boys. This study was biased towards adolescents with only 6 knees being younger than 13 .

The physical examination of an injured pediatric knee is challenging but crucial in diagnosis and treatment planning [14]. Gait and alignment should be assessed and the presence of an effusion should be sought. The examiner should palpate for tenderness over the joint line, ligamentous insertions, and the borders of the patella and retinaculum. Documentation of range of motion, patellar apprehension, varus and valgus stability should be performed. Anterior and posterior drawer should be checked along with Lachman's test and pivot shift evaluation. Most children have some inherent hyperlaxity in normal joint which the examiner should anticipate. For example, anterior knee translation decreases as children mature and is lower in boys [18].

\section{Imaging}

Radiographic evaluation includes anteroposterior, lateral, notch and skyline views of the affected knee. These
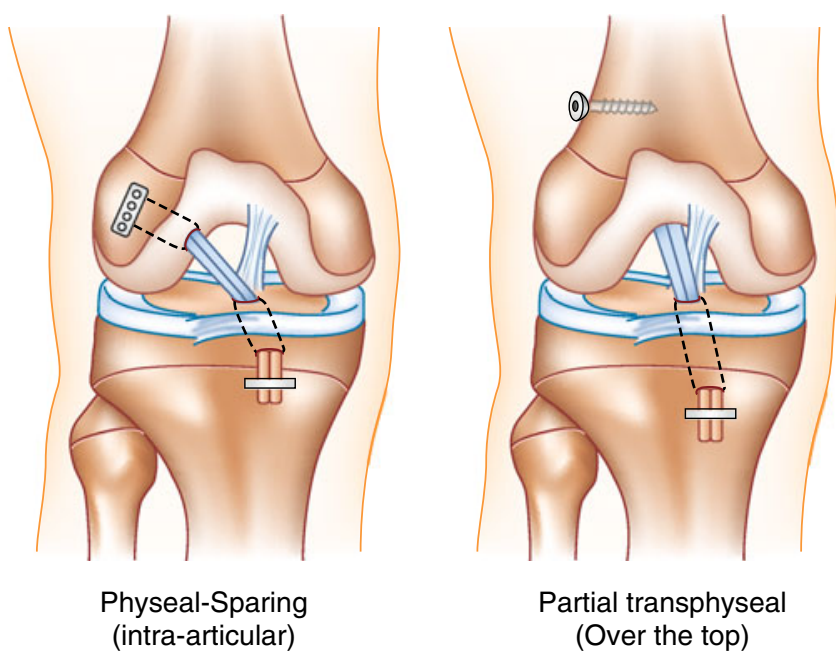

Fig. 1 Most commonly used techniques for pediatric ACL reconstruction from left to right: Intra-articular physeal-sparing technique; Partial transphyseal technique, with proximal over the top positioning

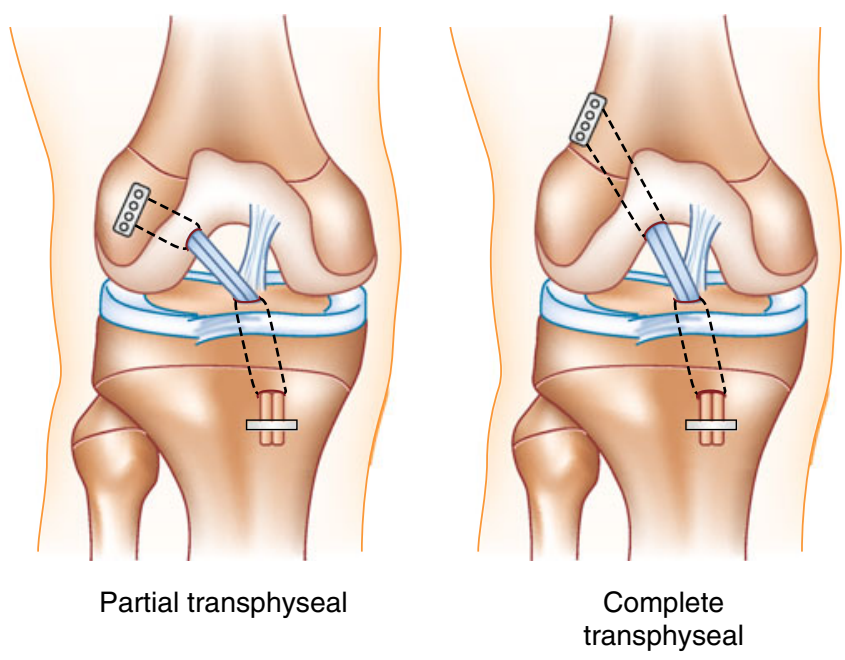

of the graft; Partial transphyseal technique, with all epiphyseal proximal tunnel drilling; and Complete transphyseal technique 
radiographs can rule out bony injuries such as tibial eminence fractures, Segond fractures, physeal injuries, osteochondral fractures and loose bodies. They also allow assessment of patellar morphology and position as well as gross assessment of physeal maturity in the adolescent patient. In addition, radiographic changes of the intercondylar notch and tibial eminences may be an indirect sign of ACL agenesis, which needs to be ruled out before planning a reconstruction [19].

Magnetic resonance imaging (MRI) is a useful tool for diagnosis and treatment planning in the skeletally immature patient. However, MRI has limitations and should be used with some restraint as it has been shown to be no better than clinical examination by an experienced surgeon for the diagnosis of ACL and meniscus tears $[20,21]$. Less experience with interpretation of pediatric knee MRI contributes to the difficulty making accurate diagnoses as does the high incidence of partial injuries and the changes in morphology with age. For example, Kim et al. [22] demonstrated an increase of the angle between the ACL and the tibial plateau in both the coronal and sagittal planes as the child matures. Classic MRI findings of discontinuity of ACL fibers and lateral compartment bone contusions may not be as reliable in the pediatric population as in the adult population [23, 24]. In contrast to the above reports, recent evidence suggests that the accuracy is excellent in pediatric patients [24, 25]. Any concern for the presence of a complex knee injury that may change management should warrant consideration of MRI evaluation.

\section{Natural history of the ACL deficient knee}

The risk of growth disturbance related to physeal injury has led surgeons to advocate for delayed treatment of ACL injury in the skeletally immature. With an ACL deficient knee return to an active lifestyle often results in repeated instability episodes and progressive intraarticular damage $[6,8,9 \cdot, 10,11,26,27]$. Many children find it difficult to modify recreational activities sufficiently to avoid instability episodes. Aichroth et al. [8] attempted to manage 33 children and adolescents with a mean age of 12.5 years with rehabilitation, bracing and activity modification. Ten of the children required stabilization due to increasing instability. At 72 months post-injury those managed nonoperatively required significant reduction in activity, had significant decline in their Lysholm score and many had signs of early osteoarthritis. McCarroll et al. [26] found in 38 patients with ACL injuries followed for 4.2 years that 27 developed signs of meniscal injury and 37 of 38 had instability symptoms. Mizuta et al. [27] presented similar results in 18 patients managed non-operatively. At 36 month follow-up 17 complained of instability, all 18 complained of pain and only 1 returned to previous activity levels.

\section{Non-operative treatment}

Non-operative management as the definitive treatment of ACL deficiency in children and adolescents is not recommended as it leads to intra-articular injury, functional impairment and joint degeneration $[6,7,11]$. Because ACL injury in children tends to occur within 6-12 months of skeletal maturity [28] some authors advocate short term non-operative management with delayed, anatomic ACL reconstruction. Woods and O'Connor [28] employed a protocol to delay reconstruction that included rehabilitation, absolute avoidance of pivoting activities and full time brace wear while awake. Compared to a control group who received acute reconstructions, they reported no increase in intra-articular injuries despite a mean delay of 70 weeks until ACL reconstruction. Moksnes et al. [29] delayed ACL reconstruction in young patients until skeletal maturity with extensive rehabilitation and return to activities as tolerated with brace wear. They reported $65 \%$ of children were able to return to previous activities without reconstruction and only $9.5 \%$ suffered a secondary meniscus injury.

Delaying surgery until physeal closure allows an anatomical adult-like reconstruction with which the surgeon is likely more familiar. Despite the acceptable results of two reported studies, the patient and parents must be extensively counseled regarding the requirements of rehabilitation, activity modifications and the risk of further injury with instability episodes if a delay in reconstruction is considered.

\section{Operative treatment}

The goal of ACL reconstruction in children and adolescents is to provide long term stability to the knee while minimizing the risk of growth disturbance. Current options for ACL reconstruction include physeal sparing procedures, partial transphyseal procedures and complete transphyseal procedures (Fig. 1).

\section{Growth effects of physeal damage}

Although there are very few human studies documenting growth disturbances, many animal studies have attempted to define the strategies to best avoid growth complications when operating around open physes. In vitro and in vivo research demonstrates that physeal arrest can be treated by resection of the physeal bar and interposition of soft tissue $[30,31]$ or cement graft [32]. It is known that placement of bridging bone graft across a physis arrests growth [33], 
however if a defect of the same size is left empty growth is minimally affected [33].

Studies of ACL reconstruction in immature animals have increased the understanding of the effects of soft tissue interposition on physeal disturbance. It has been shown in animal models that: 1) ACL tunnels filled with soft tissue grafts are less likely to form physeal bars and cause shortening or angular deformity compared with empty tunnels [34, 35], irrespective of the tunnel size [35]; 2) overtensioning of transphyseal grafts in skeletally immature individuals may predispose to growth disturbance [36]; 3) the amount of physeal damage and the presence of bioabsorbable devices at the level of the physis affect the chance of growth arrest [37-39]. Animal studies suggest that damage to less than $5 \%$ of the physis is unlikely to affect growth but that injury to 7-9\% of the physis may cause a growth arrest [37-39]. It has been estimated that an $8 \mathrm{~mm}$ femoral tunnel in a 12 year old causes injury to $3-4 \%$ of the surface area of the physis [39].

\section{Physeal sparing techniques}

Numerous ACL reconstruction techniques have been developed for use in children to avoid the physis and decrease the risk of growth disturbance. In the 1990's Brief [40] described a technique in which the hamstrings were left attached to the pes anserinus and passed over the anterior tibia, under the anterior horn of the meniscus and fixed on the femur in an over the top position. Six of 9 patients were satisfied at 3 years and returned to their previous level of sport participation. Parker et al. [41] performed a similar procedure but made a trough in the tibial epiphysis to allow a more isometric reconstruction. Four of 5 patients returned to their previous level of competition and there were no growth disturbances noted in either study. Kocher et al. [42] reported outcomes at an average of 5.3 years postoperatively on 44 Tanner stage 1 or 2 patients who underwent a combined intra and extraarticular extraphyseal ACL reconstruction using iliotibial band autograft. They reported overall excellent functional outcomes with no leg length discrepancies and two graft failures at 4.7 and 8.3 years postoperatively.

Transepiphyseal reconstructions were developed to allow more anatomically accurate ACL reconstructions in skeletally immature patients, while minimizing harm to the physis. Guzzanti et al. [43] first described a technique in which the graft is passed through an epiphyseal tibial tunnel and looped around a staple placed in the ACL footprint on the femur. Anderson [44] reported a series of 12 patients who underwent reconstruction with an epiphyseal tibial tunnel and a femoral tunnel drilled with fluoroscopic aid through the lateral femoral epiphysis. Neither of these studies reported any growth disturbance and both reported excellent functional outcomes and ligament laxity testing with no failures.

In 2011 Kennedy et al. [45•] performed a biomechanical analysis of 3 pediatric ACL reconstruction techniques: allepiphyseal, transtibial over-the-top and iliotibial band physeal-sparing. They reported that the iliotibial band physeal-sparing reconstruction best restored stability to the knee but found that it overconstrained the knee to rotational forces. The all-epiphyseal technique was unable to restore anteroposterior translation to intact values at flexion angles of $30^{\circ}$ or greater and rotational control was also inferior to the intact knee at angles of $15^{\circ}$ or greater. Some concern exists in comparing the allepiphyseal technique performed in this study to Anderson's data as the anteroposterior translation side-to-side difference was found to be $1.5+/-1.1 \mathrm{~mm}$ in Anderson's in-vivo study versus $5.1+/-2.3 \mathrm{~mm}$ in this cadaveric in-vitro research.

The evolution of physeal-sparing techniques to more anatomic reconstructions shows promise, however, the literature still includes few patients and the techniques are technically challenging with little room for error.

\section{Partial transphyseal techniques}

Partial transphyseal techniques have been reported as a means of placing a more isometric tibial graft and avoiding the distal lateral femoral physis. The graft is typically placed through a transphyseal tibial tunnel and then is fixed to the femur in an over-the-top position [46-48], or in epiphyseal tunnels [9, 49].

In 1986, Lipscomb and Anderson [49] reported a technique with transphyseal tibial tunnels and epiphyseal femoral tunnels drilled with fluoroscopic guidance through the ACL footprint. They reported excellent stability testing and return to sporting activities. There was one leg length discrepancy due to accidental physeal stapling during graft fixation. Andrews et al. [46] reported a series of 8 patients treated with transphyseal tibial tunnels and over-the-top femoral placement. They reported 6 excellent, 1 good and 1 fair result at an average of 58 months follow-up with no significant leg length discrepancies. Lo et al. [48] reported the results of 5 patients treated with transtibial, over-the-top ACL reconstructions. Four out of 5 patients reported excellent results and stability of the knee. There were no significant growth abnormalities.

In 2009, Henry et al. [9•] compared two groups of ACL injured children. The majority of Group 1 underwent subacute ACL reconstruction with a transphyseal tibial tunnel and an anatomic epiphyseal femoral tunnel. Five of 29 underwent reconstruction with a physeal sparing iliotibial band autograft passed over-the-top then through a transphyseal tibial tunnel. Group 2 was managed conservatively until skeletal maturity and then underwent standard bone- 
tendon-bone reconstruction. Group 2 had significantly more medial meniscus tears at the time of operation and had significantly lower functional and clinical results at final follow-up. There were no growth abnormalities in either group. The authors recommend early ACL reconstruction.

\section{Transphyseal techniques}

Complete transphyseal ACL reconstruction offers the benefit of being very similar to adult ACL reconstruction and therefore familiar to most surgeons. It allows isometric tunnel placement which may improve graft longevity and knee function. Violation of the physis creates concern that the incidence of growth disturbance may increase, especially in children who are very skeletally immature.

A number of series' report transphyseal ACL reconstructions using a variety of grafts and fixation devices with good outcomes in pediatric patients and little risk of growth disturbance [8, 10, 50-55], [56•], [57], [58•]. Aronowitz et al. [50] used Achilles tendon allograft with 9-10 mm tunnels in patients with a skeletal age of at least 14. Follow-up at skeletal maturity revealed an average Lysholm score of 97, no instability and no growth disturbances. They concluded that transphyseal ACL reconstruction can safely be used in patients with a bone age of at least 14 years. In a 2002 report of 10 transphyseal bone-tendon-bone ACL reconstructions 5 of 6 boys had no axillary hair and all of the girls were premenarchal [53]. During fixation, the surgeon was careful to secure the bone plug proximal to the femoral physis and distal to the tibial physis. At final follow up there were no growth abnormalities and the average Lysholm score was 95 . The authors suggest that intra-articular, transphyseal ACL reconstructions can be performed safely with careful attention to bone plug and fixation placement.

Recently, a number of publications have reported the results of transphyseal quadrupled hamstring autogenous ACL reconstructions in skeletally immature patients [51, 52, 55, 56• 57, 58•]. Kocher et al. [55] reported 61 cases in Tanner stage III or IV patients in which the graft was fixed to the femur with an Endobutton (Smith \& Nephew, Andover, MA, USA) and tibial fixation was with an interference screw. The arthroscope was placed in the tibial tunnel prior to fixation to ensure the physis was at least $25 \mathrm{~mm}$ proximal to the aperture. Respective, mean IKDC and Lysholm scores were 89.5 and 91.2, there were 2 graft failures and no growth disturbances. The authors recommend the transphyseal technique for Tanner stage III and IV adolescents. In 2010, Courvoisier et al. [52] reported ACL reconstructions of 37 patients with wide open physes with graft fixation similar to Kocher et al. [55]. The technique was the same as for adults except the femoral tunnel was placed more vertically to avoid injury to the perichondrial ring. In follow-up 28 were IKDC A and 4 were IKDC B, there were 3 graft ruptures and no growth or angular deformities. In 2009, Cohen et al. [51] performed 26 ACL reconstructions and reported mean Lysholm and International Knee Documentation Committee (IKDC) scores of 93.5 and 91.5 , respectively. Five patients were Tanner stage I or II, 21 were Tanner stage III or IV and there were no reported growth or angular deformities. Nikolaou et al. [57] reported the results of 94 transphyseal hamstring reconstructions in patients followed to skeletal maturity. Of these patients 66 were Tanner stage I or II. They reported $90 \%$ IKDC classification A or B and a median Lysholm score of 89 with no growth or angular deformities. Finally, Liddle et al. [56•] published a series of 17 Tanner stage I or II children managed with transphyseal ACL reconstructions. Mean Lysholm scores were 97.5 but there was one rerupture and one asymptomatic $5^{\circ}$ valgus deformity without growth arrest. All five studies concluded that carefully performed transphyseal ACL reconstruction with hamstring autograft was safe and effective.

A systematic review of the English literature in 2010 examined ACL reconstructions in preadolescent patients to determine if any technique is superior to another in this population [59]. The inclusion criteria for this study were: (1) chronological and bone age less than 15 for boys and 14 for girls; (2) Tanner stage I, II or III; (3) at least $10 \mathrm{~cm}$ of growth after the reconstruction. These criteria ensured that only patients with significant growth remaining were included. Only case studies were available for comparison but they found the functional results of both physeal sparing and transphyseal reconstruction to be excellent. They concluded that transphyseal reconstruction techniques are safe for Tanner stage II and III patients but there is insufficient evidence to support or discourage its use in Tanner stage I patients.

Reports of complications of ACL reconstruction in skeletally immature patients are rare and most of the documented growth complications are attributable to surgeon error such as placement of a fixation device across a growth plate [5, 60-62]. Kocher et al. [60] surveyed the Herodicus Society and ACL study group and reported 15 cases of growth disturbance. Three were cases of tibial recurvatum due to staples or sutures across the apophysis of the tibial tuberosity and nine were distal femoral valgus deformities due to placement of staples, screws, pins or bone plugs across the physis. Limb overgrowth can rarely occur with reports of a $1.5 \mathrm{~cm}$ limb length discrepancy requiring a shoe lift [10], overgrowth requiring ipsilateral epiphysiodesis $[60,63]$, and temporary tibia valga that spontaneously corrected [63].

A recent meta-analysis of the literature included 941 physeal sparing or transphyseal reconstructions and found that the overall incidence of growth abnormalities was $2.1 \%$ and rerupture rate was $3.8 \%$ [64]. They also reported that 
physeal-sparing techniques increased risk of growth abnormalities $(1.9 \%$ versus $5.8 \%)$ but cautioned that the uncontrolled nature of the case series' included in the study makes interpretation of the results difficult. Also, grouping all physeal-sparing techniques together for statistical comparison is of questionable benefit given the heterogeneity of the group as a result of the numerous techniques included.

\section{Authors' preferred treatment}

The authors usually recommend reconstruction for pediatric ACL injuries, in order to reduce recurrent instability episodes and further damages to the knee joint. Pediatric patients can be considered as high level athletes, who are rarely compliant with strict activity modifications. An adult type ACL reconstruction is indicated in females over 15 years old and males over 16 . In younger patients, the authors usually prefer a partial transphyseal technique with proximal over-the-top positioning of the semitendinosus only graft. Acute repair using a pull-out technique, with or without augmentation, can be considered in case of proximal and distal avulsion of the ACL, both in the multiligament knee and isolated ACL injuries.

General rules that should be followed with any pediatric ACL reconstruction technique include: 1) avoid overtensioning the graft; 2) transphyseal tunnels should be completely filled by soft tissue graft; 3 ) avoid bone blocks and hardware at the level of the physes; 4) the tunnels should be small $(6-7 \mathrm{~mm})$ and almost perpendicular to the physis, in order to minimize the damage to the growth plate.

\section{Conclusion}

Injuries to the ACL in the pediatric population have increased in incidence as children have placed increased demands on their bodies with early entry into competitive athletics. In a young person with an acutely injured knee careful clinical evaluation needs to be undertaken especially in the presence of an effusion. Once the diagnosis of ACL injury is made the parents and youth should be counseled on the risks and benefits of operative and non-operative therapy.

The natural history of the ACL deficient knee in children generally results in recurrent instability episodes and progressive intra-articular injury. Delayed reconstruction has been used successfully with severe limits placed on activity and full time brace wear. Most children attempting to return to high risk activities with an ACL deficiency suffer from knee instability and functional decline.

In general, ACL reconstruction is indicated with recurrent instability or when a patient is unable or unwilling to modify his or her activities to protect the knee. By definition, children are at very high risk for instability and our approach is generally to reconstruct these knees. If there is a repairable intra-articular lesion ACL reconstruction should also be recommended.

Numerous techniques have been described for the treatment of pediatric ACL injuries. Good functional results have been obtained with physeal-sparing, partial transphyseal and complete transphyseal procedures. Adolescents who are nearing the end of their growth can be managed by as an adult with a transphyseal ACL reconstruction and minimal risk of growth disturbance.

The surgical management of pediatric ACL tears in Tanner stage I or II is controversial. Case series' have shown that properly performed physeal-sparing or partial transphyseal procedures can result in excellent stability and functional results. Recently, many authors have recommended complete transphyseal procedures in children with significant growth remaining. Several studies report excellent functional outcome and knee stability in Tanner stages I and II with minimal risk of growth disturbance.

Reports of growth disturbance are rare and usually attributable to surgical error such as placement of hardware or bone plugs across the physis. Clinical and animal research suggests the risk of growth disturbance is minimized when transphyseal tunnels are kept small and completely filled with soft tissue graft. Fixation devices and bone plugs should be kept away from the physis and overtensioning of the graft should be avoided. With careful attention to surgical technique pediatric ACL reconstruction can be safe and effective. Current evidence is limited to retrospective and prospective case series so research with improved methodology is needed.

Disclosure No conflicts of interest relevant to this article were reported.

\section{References}

Papers of particular interest, published recently, have been highlighted as:

- Of importance

1. Micheli LJ, Foster TE. Acute knee injuries in the immature athlete. AAOS Instr Course Lect. 1993;42:473-81.

2. Mohtadi N, Grant J. Managing anterior cruciate ligament deficiency in the skeletally immature individual: a systematic review of the literature. Clin J Sport Med. 2006;16:457-64.

3. Andrish JT. Anterior cruciate ligament injuries in the skeletally immature patient. Am J Orthop. 2001;30:103-10.

4. DeLee JC, Curtis R. Anterior cruciate ligament insufficiency in children. Clin Orthop Relat Res. 1983;172:112-8.

5. Lipscomb AB, Anderson AF. Tears of the anterior cruciate ligament in adolescents. J Bone Joint Surg Am. 1986;68:19-28. 
6. Graf BK, Lange R, Fujisaki CK, Landry GL, Saluja RK. Anterior cruciate ligament tears in skeletally immature patients: meniscal pathology at presentation and after attempted conservative treatment. Arthroscopy. 1992;8:229-33.

7. Kannus P, Jarvinen M. Knee ligament injuries in adolescents: eight year follow-up of conservative management. J Bone Joint Surg Br. 1988;70:772-6.

8. Aichroth PM, Patel DV, Zorrilla P. The natural history and treatment of rupture of the anterior cruciate ligament in children and adolescents: a prospective review. J Bone Joint Surg Br. 2002;84:38-41.

9. - Henry J, Chotel F, Chouteau J, et al.: Rupture of the anterior cruciate ligament in children: early reconstruction with open physes or delayed reconstruction to skeletal maturity? Knee Surg Sports Traumatol Arthrosc 2009, 17:748-755. Retrospective comparison of 2 groups of patients with open physes. Group 1 managed with acute ACL reconstruction and group 2 managed with ACL reconstruction at skeletal maturity. Results indicate higher number of meniscal tears and meniscectomies and lower subjective IKDC scores in group 2.

10. McIntosh AL, Dahm DL, Stuart MJ. Anterior cruciate ligament reconstruction in the skeletally immature patient. Arthroscopy. 2006;22:1325-30.

11. Pressman AE, Letts RM, Jarvis JG. Anterior cruciate ligament tears in children: an analysis of operative versus nonoperative treatment. J Pediatr Orthop. 1997;17:505-11.

12. Larsen MW, Garrett Jr WE. Delee JC, Moorman CT 3rd: surgical management of anterior cruciate ligament injuries in patients with open physes. J Am Acad Orthop Surg. 2006;14:736-44.

13. Diméglio A. Growth in pediatric orthopaedics. In: Morrissey RT, Weinstein SL, editors. Lovell and winter's pediatric orthopaedics. 5th ed. Philadelphia: Lippincott; 2001. p. 33-63.

14. Stanitski CL. Correlation of arthroscopic and clinical examinations with magnetic resonance imaging findings of injured knees in children and adolescents. Am J Sports Med. 1998;26:2-6.

15. Boden BP, Dean GS, Feagin JA, Garret WE. Mechanisms of anterior cruciate ligament injury. Orthop Jun. 2000;23:573-8.

16. Stanitski CL, Harvell JC, Fu F. Observations on acute knee hemarthrosis in children and adolescents. J Pediatr Orthop. 1993;13:506-10.

17. Luhmann SJ. Acute traumatic knee effusions in children and adolescents. J Pediatr Orthop. 2003;23:199-202.

18. Hinton RY, Rivera VR, Pautz MJ, Sponseller PD. Ligamentous laxity of the knee during childhood and adolescence. J Pediatr Orthop. 2008;28:184-7.

19. Manner HM, Radler C, Ganger R, Grill F. Dysplasia of the cruciate ligaments: radiographic assessment and classification. J Bone Joint Surg. 2006;88:130-7.

20. Kocabey Y, Tetik O, Isbell WM, Atay A, Johnson DL. The value of clinical examination versus magnetic resonance imaging in the diagnosis of meniscal tears and anterior cruciate ligament rupture. Arthroscopy. 2004;20:696-700.

21. Kocher MS, DiCanzio J, Zurakowski D, Micheli LJ. Diagnostic performance of clinical examination and selective magnetic resonance imaging in the evaluation of intraarticular knee disorders in children and adolescents. Am J Sports Med. 2001;29:292-6.

22. Kim HK, Laor T, Shire NJ, Bean JA, Dardzinski BJ. Anterior and posterior cruciate ligaments at different patient ages: MR imaging findings. Radiology. 2008;247:826-35.

23. Coursey Jr RL, Jones EA, Chaljub G, et al. Prospective analysis of uncomplicated bone bruises in the pediatric knee. Emerg Radiol. 2006;12:266-71.

24. Lee K, Siegel MJ, Lau DM, et al. Anterior cruciate ligament tears: MR imaging-based diagnosis in a pediatric population. Radiology. 1999;213:697-704.
25. Major NM, Beard Jr LN, Helms CA. Accuracy of MR imaging of the knee in adolescents. AJR Am J Roentgenol. 2003;180:17-9.

26. McCarroll JR, Shelbourne KD, Porter DA, Retting AC, Murray S. Patellar tendon graft reconstruction for mid-substance anterior cruciate ligament rupture in junior high school athletes. An algorithm for management. Am J Sports Med. 1994;22:478-84.

27. Mizuta H, Kubota K, Shiraishi M, et al. The conservative treatment of complete tears of the anterior cruciate ligament in skeletally immature patients. J Bone Joint Surg Br. 1995;77:890-4.

28. Woods GW, O'Connor DP. Delayed anterior cruciate ligament reconstruction in adolescents with open physes. Am J Sports Med. 2004:32:201-10.

29. Moksnes H, Engebretsen L, Risberg MA. Performance-based functional outcome for children 12 years or younger following anterior cruciate ligament injury: a two to nine-year follow-up study. Knee Surg Sports Traumatol Arthrosc. 2008;16:214-23.

30. Langenskiöld $\mathrm{A}$. The possibilities of eliminating premature partial closure of an epiphyseal plate caused by trauma or disease. Acta Orthop Scand. 1967;38:267-79.

31. Österman K. Healing of large surgical defects of the epiphyseal plate. Clin Orthop Relat Res. 1994;300:264-8.

32. Peterson HA. Partial growth plate arrest and its treatment. J Pediatr Orthop. 1984;4:246-58.

33. Campbell CJ, Grisolia A, Zanconato G. The effects produced in the cartilaginous epiphyseal plate of immature dogs by experimental surgical traumata. J Bone Joint Surg Am. 1959;41:1221-42.

34. Stadelmaier DM, Arnoczky SP, Dodds J, Ross H. The effect of drilling and soft tissue grafting across open growth plates: a histologic study. Am J Sports Med. 1995;23:431-5.

35. Seil R, Pape D, Kohn D. The risk of growth changes during transphyseal drilling in sheep with open physes. Arthroscopy. 2008;24:824-33.

36. Edwards TB, Greene CC, Baratta RV, Zieske A, Willis RB. The effect of placing a tensioned graft across open growth plates: a gross and histologic analysis. J Bone Joint Surg Am. 2001;83:725-34.

37. Makela EA, Vainionpaa S, Vihtonen K, Mero M, Rokkanen P. The effect of trauma to the lower femoral epiphyseal plate: an experimental study in rabbits. J Bone Joint Surg Br. 1988;70:187-91.

38. Makela EA, Vainionpaa S, Vihtonen K, et al. The effect of a penetrating biodegradable implant on the growth plate. An experimental study on growing rabbits with special reference to polydioxanone. Clin Orthop Relat Res. 1989;241:300-8.

39. Janarv PM, Wikstrom B, Hirsch G. The influence of transphyseal drilling and tendon grafting on bone growth: an experimental study in the rabbit. J Pediatr Orthop. 1998;18:149-54.

40. Brief LP. Anterior cruciate ligament reconstruction without drill holes. Arthroscopy. 1991;7:350-7.

41. Parker AW, Drez Jr D, Cooper JL. Anterior cruciate ligament injuries in patients with open physes. Am J Sports Med. 1994:22:44-7.

42. Kocher MS, Garg S, Micheli LJ. Physeal sparing reconstruction of the anterior cruciate ligament in skeletally immature prepubescent children and adolescents. J Bone Joint Surg Am. 2005;87:2371-9.

43. Guzzanti V, Falciglia F, Stanitski CL. Physeal-sparing intraarticular anterior cruciate ligament reconstruction in preadolescents. Am J Sports Med. 2003;31:949-53.

44. Anderson AF. Transepiphyseal replacement of the anterior cruciate ligament using quadruple hamstring grafts in skeletally immature patients. J Bone Joint Surg Am. 2004;86:201-9.

45. - Kennedy A, Coughlin DG, Metzger MF, et al.: Biomechanical evaluation of pediatric anterior cruciate ligament reconstruction techniques. Am J Sports Med 2011, In press. Three pediatric ACL reconstruction techniques (all epiphyseal, transtibial over-the-top, IT band extra/intraarticular physeal sparing) were compared in biomechanics lab. All epiphyseal and transtibial techniques 
improved AP translation but not to ACL intact state. IT band technique best restored AP translation but may overconstrain the knee to rotational forces.

46. Andrews M, Noyes FR, Barber-Westin SD. Anterior cruciate ligament allograft reconstruction in the skeletally immature athlete. Am J Sports Med. 1994;22:48-54.

47. Bonasia DE, Rossi R, Wolf BR, Amendola A: Pediatric anterior cruciate ligament reconstruction. Presented at 2011 American academy of orthopaedic surgeons annual meeting multimedia education center. San Diego, USA; February 15-19, 2011.

48. Lo IK, Kirkley A, Fowler PJ, Miniaci A. The outcome of operatively treated anterior cruciate ligament disruptions in the skeletally immature child. Arthroscopy. 1997;13:627-34.

49. Lipscomb AB, Anderson AF. Tears of the anterior cruciate ligament in adolescents. J Bone Joint Surg Am. 1986;68:19-28.

50. Aronowitz ER, Ganley TJ, Goode JR, Gregg JR, Meyer JS. Anterior cruciate ligament reconstruction in adolescents with open physes. Am J Sports Med. 2000;28:168-75.

51. Cohen M, Ferretti M, Quarteiro M, et al. Transphyseal anterior cruciate ligament reconstruction in patients with open physes. Arthroscopy. 2009;25:831-8.

52. Courvoisier A, Grimaldi M, Plasweski S. Good surgical outcome of transphyseal ACL reconstruction in skeletally immature patients using four-strand hamstring graft. Knee Surg Sports Traumatol Arthrosc. 2011;19:588-91.

53. Fuchs R, Wheatley W, Uribe JW, et al. Intra-articular anterior cruciate ligament reconstruction using patellar tendon allograft in the skeletally immature patient. Arthroscopy. 2002;18:824-8.

54. Gaulrapp HM, Haus J. Intraarticular stabilization after anterior cruciate ligament tear in children and adolescents: results 6 years after surgery. Knee Surg Sports Traumatol Arthrosc. 2006;14: 417-24.

55. Kocher MS, Smith JT, Zoric BJ, Micheli LJ. Transphyseal anterior cruciate ligament reconstruction in skeletally immature pubescent adolescents. J Bone Joint Surg Am. 2007;89:2632-9.

56. - Liddle AD, Imbuldeniya AM, Hunt DM: Transphyseal reconstruction of the anterior cruciate ligament in prepubescent children. J Bone Joint Surg Br 2008, 90:1317-1322. 17 Tanner 1 and 2 patients underwent transphyseal ACL reconstruction with quadrupled hamstring autograft. Lysholm, IKDC, Tegner activity scores and KT1000 measurements were excellent. There was one rerupture and one asymptomatic mild valgus deformity.

57. Nikolaou P, Kalliakmanis A, Bousgas D, Zourntos S. Intraarticular stabilization following anterior cruciate ligament injury in children and adolescents. Knee Surg Sports Traumatol Arthrosc. 2011. doi:10.1007/s00167-010-1375-y.

58. - Streich NA, Barie A, Gotterbarm T, Keil M, Schmitt H: Transphyseal reconstruction of the anterior cruciate ligament in prepubescent athletes. Knee Surg Sports Traumatol Arthrosc 2010, 18:1481-1486. Comparison between nonoperative and operative management (Transphyseal hamstring autograft) in Tanner 1 and 2 patients was performed. Early reconstructions were performed on those patients with other intra-articular pathology (meniscus tear, osteochondral fracture), whereas nonoperative management was undertaken in those patients with isolated ACL tears. Outcome scores were significantly improved in the operative groups. $58 \%$ on the nonoperative group underwent sub-acute ACL reconstruction due to ongoing instability and $86 \%$ of this group had meniscal tears at the time of operation.

59. Kaeding CC, Flanigan D, Donaldson C. Surgical techniques and outcomes after anterior cruciate ligament reconstruction in preadolescent patients. Arthroscopy. 2010;26:1530-8.

60. Kocher MS, Saxon HS, Hovis WD, Hawkins RJ. Management and complications of anterior cruciate ligament injuries in skeletally immature patients: survey of the Herodicus Society and the ACL Study Group. J Pediatr Orthop. 2002;22:452-7.

61. Barber FA, Sanders JO, Clark R. Anterior cruciate ligament reconstruction in the skeletally immature high performance athlete. What to do and when to do it? Arthroscopy. 2000;16:391-4.

62. Koman JD, Sanders JO. Valgus deformity after reconstruction of the anterior cruciate ligament in a skeletally immature patient. A case report. J Bone Joint Surg. 1999;81:711-5.

63. Chotel F, Henry J, Seil R, et al. Growth disturbances without growth arrest after ACL reconstruction in children. Knee Surg Sports Traumatol Arthrosc. 2010;18:1496-500.

64. Frosch KH, Stengel D, Brodhun T, et al. Outcomes and risks of operative treatment of rupture of the anterior cruciate ligament in children and adolescents. Arthroscopy. 2010;26:1539-50. 